\title{
Improved near-infrared sensitivity of a back-side illuminated image sensor with a metal reflector
}

\author{
Tetsuya Ariyoshi ${ }^{\mathrm{a})}$, Shozo Morita, Akiyoshi Baba, \\ and Yutaka Arima \\ Center for Microelectronic Systems, Kyushu Institute of Technology, 680-4 \\ Kawazu, Iizuka, Fukuoka 820-8502 Japan \\ a)ariyoshi@cms.kyutech.ac.jp
}

\begin{abstract}
To improve the near-infrared sensitivity of an image sensor that has a conventional pixel structure, we made use of the metal wiring layer as a reflector in addition to the method of back-side illumination from the etched back surface of the sensor. We fabricated an image sensor that has a remaining silicon substrate thickness of $32 \pm 4 \mu \mathrm{m}$ and evaluated the spectral sensitivity. For $830 \mathrm{~nm}$ light, the sensitivity is about the same as for front-side illumination. For a wavelength of $970 \mathrm{~nm}$, the sensitivity was 2.2 times as high as for front-side illumination. The image sensor with reflector has about $30 \%$ higher sensitivity than without the reflector.
\end{abstract}

Keywords: near-infrared light, higher sensitivity, back-side illumination, Deep-RIE, metal reflector

Classification: Optoelectronics, Lasers and quantum electronics, Ultrafast optics, Silicon photonics, Planar lightwave circuits

\section{References}

[1] W. C. Dash and R. Newman, "Intrinsic Optical Absorption in SingleCrystal Germanium and Silicon at 77K and 300K," Physical review, vol. 99, no. 4, pp. 1151-1155, Aug. 1955.

[2] S. Iwabuchi, Y. Maruyama, Y. Ohgishi, M. Muramatsu, N. Karasawa, and T. Hirayama, "A Back-Illuminated High-Sensitivity Small-Pixel Color CMOS Image Sensor with Flexible Layout of Metal Wiring," ISSCC Dig. Tech. Papers, pp. 302-303, 2006.

[3] H.-q. Jiang, X. Yao, J. Che, and M.-q. Wang, "The photoluminescence and optical constant of $\mathrm{ZnSe} / \mathrm{SiO}_{2}$ thin films prepared by sol-gel process," Materials Research Bulletin, vol. 41, pp. 2349-2356, 2006.

[4] E. D. Palik, "Handbook of Optical Constants of Solids," Academic Press Inc., pp. 398-399, 565, 1985. 


\section{Introduction}

Conventional visible light image sensors that use silicon photodiodes can detect near-infrared light up to a wavelength of $1.1 \mu \mathrm{m}$. This kind of image sensor can be produced at low cost because special processes such as cooling are not necessary. Accordingly, the conventional type of image sensor is also used for night-vision cameras that employ near-infrared illumination. Nevertheless, using the conventional pixel structure is problematic because the near-infrared sensitivity is low compared to the sensitivity to visible light. This low sensitivity is caused by the low rate of absorption of silicon for long wavelength light [1]. To compensate that low sensitivity, the output power of the near-infrared light source must be sufficiently high, but the size and power consumption of the equipment has practical limits. An etched back-side illumination CMOS image sensor for visible light has already been developed and its effectiveness has been demonstrated [2].

Our objective is to increase the sensitivity of the conventional image sensor structure to near-infrared light. In the work reported here, we confirmed the effectiveness of using the metal wiring layer as a reflector for reflecting

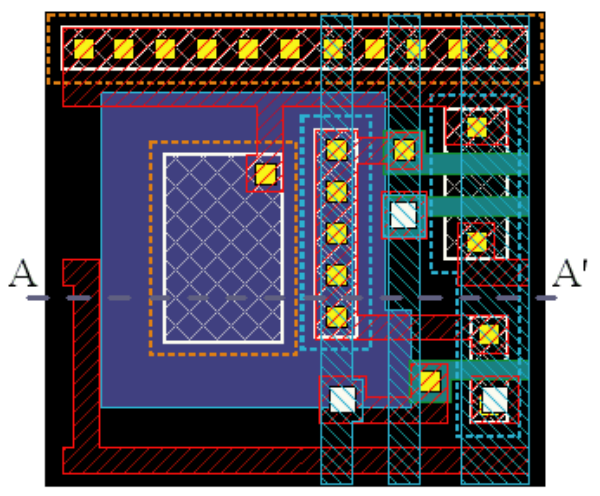

(a)

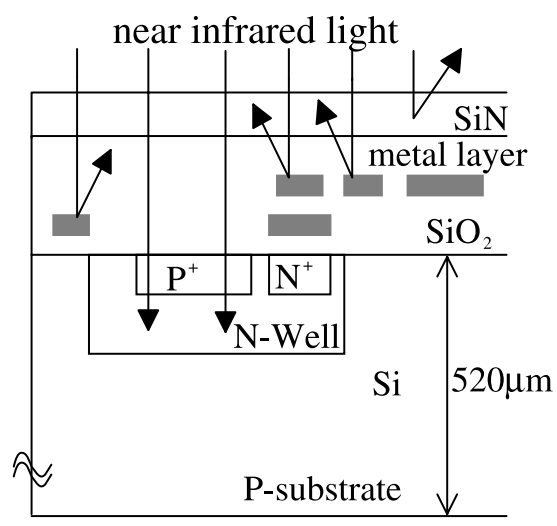

(c)

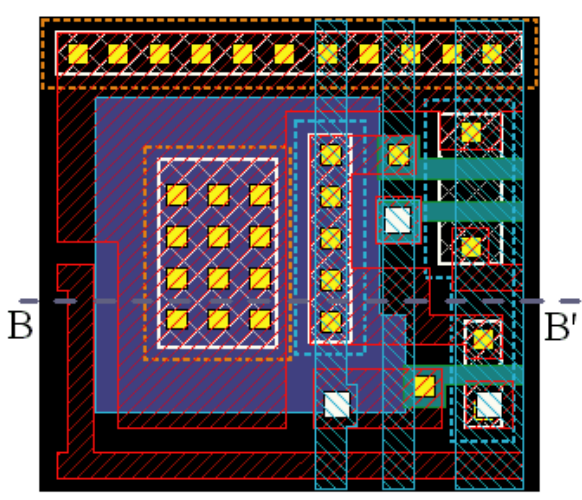

(b)

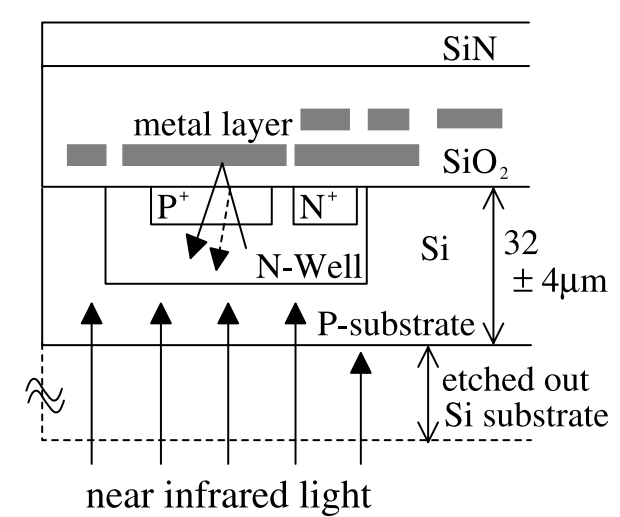

(d)

Fig. 1. Pixel structure: (a) Front-side illumination pixel layout, (b) Etched back-side illumination pixel layout, (c) A-A' cross-section structure, (d) B-B' cross-section structure. 
near-infrared light in addition to the method of near-infrared illumination from the back-side. The reflector serves to increase the opportunity for absorption of near-infrared light by extending the optical path of the nearinfrared light within the silicon. We applied these two methods to increase the sensitivity of the image sensor to near-infrared light. To test the effectiveness of the proposed near-infrared light image sensor, we fabricated a test device and evaluated the spectral sensitivity for near-infrared light.

\section{Pixel structure}

A test device is fabricated by austriamicrosystems AG with $0.35 \mu \mathrm{m}$ CMOS 1poly, 3-metal and 3.3-Volt process. The pixel structure of the fabricated test device is shown in Fig. 1, where (a) is the conventional front-side illumination pixel layout and (c) is the A-A' cross section of that structure. The pixel layout for the etched back-side illumination is shown in Fig. 1 (b), and (d) in the figure is the cross section structure from B to B'. The pixel size of the testfabricated image sensor is $9.0 \times 9.0 \mu \mathrm{m}^{2}$. LOCOS is adopted for isolation. The photodiodes are composed of a P-substrate and N-Well. Furthermore, $\mathrm{P}^{+} \mathrm{S} / \mathrm{D}$ is formed within the N-Well, and the photodiode is configured in the same way. This image sensor has a $\mathrm{SiN}$ front-side protection layer and a $\mathrm{SiO}_{2}$ interlayer insulation layer.

The metal wiring in the conventional front-side illumination scheme reduces the pixel opening area ratio, so the amount of incident light that reaches the photodiode is limited. With the etched back-side illumination shown in Fig. 1 (d) on the other hand, there is no metal wiring layer on the back-side to block incident light, so the opening area ratio is larger. The pixel opening area ratio as seen from the back-side is 1.6 times as large as seen from the front-side. Furthermore, the proposed structure employs the metal wiring layer on the front-side of the pixel as a reflector, thereby extending the path of the light through the silicon and increasing the opportunity for light absorption by the silicon. The metal layer we used here is aluminum. As shown in Fig. 1(d), the near-infrared light is reflected by the metal layer, but some is reflected at the $\mathrm{Si} / \mathrm{SiO}_{2}$ interface. The total reflectivity that takes multiple reflection into account can be calculated using the complex refractive indices of $\mathrm{Si}, \mathrm{SiO}_{2}$ and $\mathrm{Al}[3,4]$. The derived reflectivity of near-infrared light is $89.6 \%$ at the wavelength of $830 \mathrm{~nm}$ and $80.3 \%$ at $970 \mathrm{~nm}$. Without the reflector, only the $\mathrm{Si} / \mathrm{SiO}_{2}$ interface contributes to the reflection, and the respective reflectivities are then $18.8 \%$ and $18.0 \%$. Using the reflector raises the reflectivity for near-infrared light by a factor of 4 or 5 .

\section{Test chip}

The test chip fabricated by the $0.35 \mu \mathrm{m}$ CMOS 1-poly, 3-metal and 3.3-Volt process is shown in Fig. 2, where (a) is a photograph of the front-side of the chip. The chip dimensions are $4.2 \mathrm{~mm} \times 3.3 \mathrm{~mm}$. The silicon substrate thickness was $520 \mu \mathrm{m}$ before the back-side etching. On the test chip are arranged four types of image sensors that differ in photodiode structure. The 


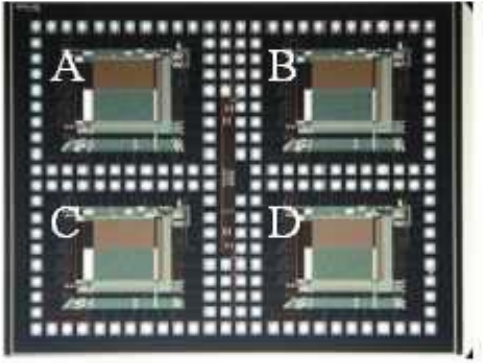

(a)

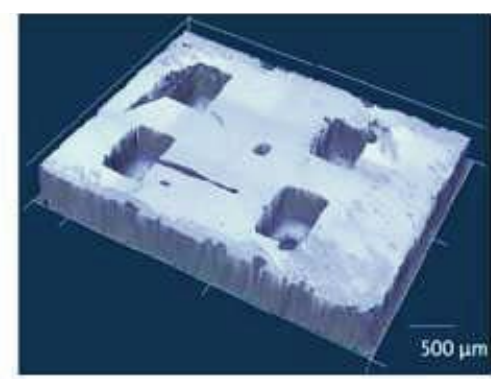

(b)

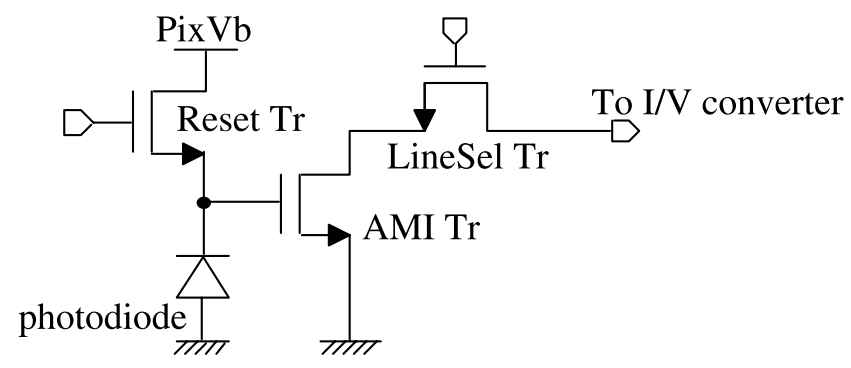

(c)

Fig. 2. Near-infrared image sensor test chip: (a) Photograph of chip front-side surface (b) Laser microscope image of the back-side of a back-etched chip (c) Pixel circuit configuration.

pixels of image sensor A in Fig. 2 (a) uses a photodiode formed of an N-Well and P-sub. The pixels of image sensor B use the same photodiode as A, but have twice as many charge-collecting contacts. The pixels of image sensor $\mathrm{C}$ use a photodiode that consists of an N-Well sandwiched between a P-sub and a $\mathrm{P}^{+} \mathrm{S} / \mathrm{D}$. The pixels of image sensor $\mathrm{D}$ use the same photodiode as $\mathrm{C}$, but have twice as many charge-collecting contacts. The image sensors consist of a 64 by 64 grid of pixels. The bottom half $(64 \times 32)$ of each has a metal layer reflector. Accordingly, the effectiveness of the reflector can be tested by comparing the near-infrared light back-side illumination characteristics of the upper and lower halves of the image sensors.

After fabricating the test chip, the etching was performed. To prevent damage to the finished image sensors, we used low-temperature RIE (Reactive Ion Etching) to etch the back-side of the silicon substrate. A laser microscope photograph of the back-side of a chip after etching is shown in Fig. 2 (b). The back-side etching was done on the left halves of each image sensor $(32 \times 64)$. The silicon thickness in the etched areas is $32 \pm 4 \mu \mathrm{m}$ and the etched areas are $500 \times 800 \mu \mathrm{m}^{2}$. The upper right parts of the image sensors $(32 \times 32)$ can be used to evaluate the pixel photosensitivity for front-side illumination. The pixel circuit configuration is shown in Fig. 2 (c). The bias voltage, PixVb, gives the photodiode reset voltage, which is $2.0 \mathrm{~V}$ in this evaluation. The cathode potential of the photodiode decreases as a result of photoelectric conversion. AMI (Amplified MOS Imager) transistor

(c) IEICE 2009

DOI: $10.1587 /$ elex.6.341 Received January 30, 2009 Accepted February 06, 2009 Published March 25, 2009 


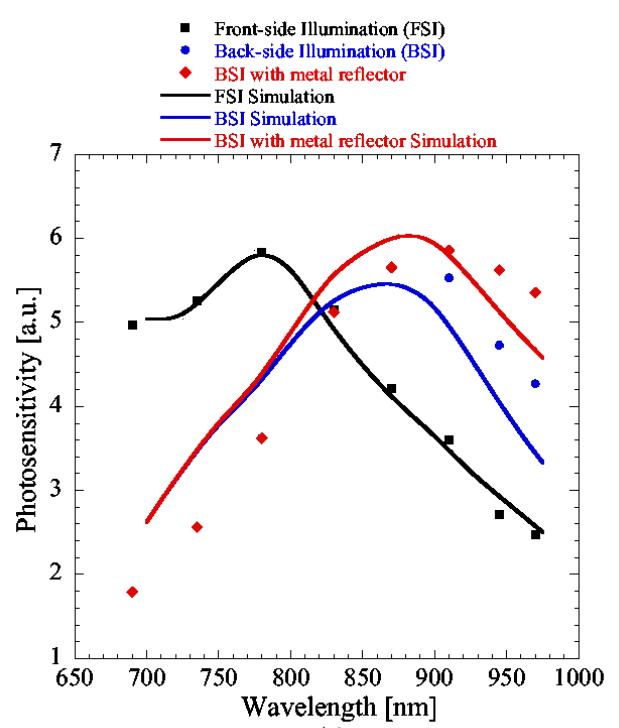

(a)

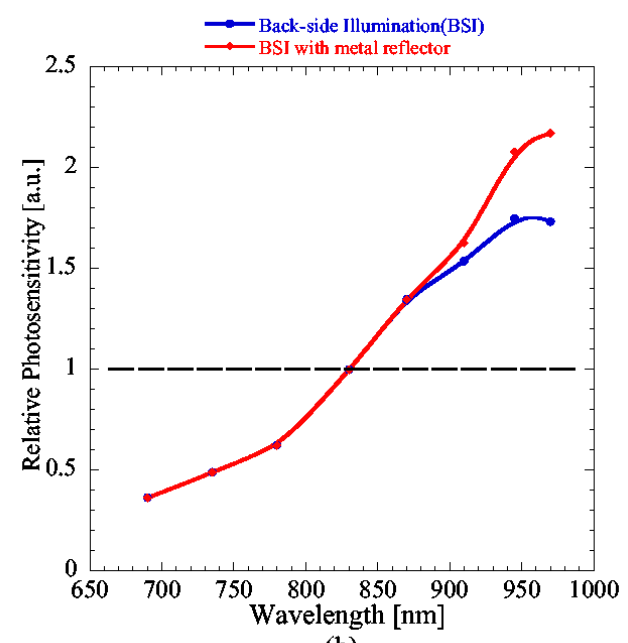

(b)

Fig. 3. The results of photosensitivity measurements: (a) Photosensitivity dependence on wavelength. The solid lines show the results of the optical simulation. (b) Relative photosensitivity of the back-side illumination compared to the front-side illumination.

converts the decrease of the cathode potential into the current. I/V converter converts this current into the output voltage.

\section{Evaluation of the photosensitivity characteristic}

We evaluated the dependence of photosensitivity on wavelength for image sensor D, which uses a pixel structure that can suppress the variance of the dark current. We performed experiments in which the front-side and the back-side of the test chip were illuminated with light from eight types of lightemitting diodes and the photosensitivity was measured for each wavelength.

The light-emitting diodes that we used had peak wavelengths of $690 \mathrm{~nm}$ $(\Delta \lambda=20 \mathrm{~nm}), 735 \mathrm{~nm}(\Delta \lambda=30 \mathrm{~nm}), 780 \mathrm{~nm}(\Delta \lambda=30 \mathrm{~nm}), 830 \mathrm{~nm}(\Delta \lambda=$ $35 \mathrm{~nm}), 870 \mathrm{~nm}(\Delta \lambda=35 \mathrm{~nm}), 910 \mathrm{~nm}(\Delta \lambda=50 \mathrm{~nm}), 945 \mathrm{~nm}(\Delta \lambda=45 \mathrm{~nm})$, and $970 \mathrm{~nm}(\Delta \lambda=55 \mathrm{~nm})$, where $\Delta \lambda$ is emission spectrum at half width. The results of the photosensitivity measurements are shown in Fig. 3, where (a) shows the measured dependence of photosensitivity on wavelength for front-side illumination and back-side illumination and (b) shows the relative photosensitivity of the back-side illumination compared to the front-side illumination. The solid lines in Fig. 3 (a) represent the optical simulation results. The results for measured photosensitivity with front-side illumination show that the peak sensitivity near $780 \mathrm{~nm}$ corresponds to the peak transparency $97 \%$ of the SiN front-side protection layer near $780 \mathrm{~nm}$ near-infrared light. The discrepancy between the simulation and the actual measurements for back-side illumination may be the effect of etching residue adhering to the back-side silicon. 
When the wavelength of the illuminating light is $830 \mathrm{~nm}$ or longer, sensitivity is higher for back-side illumination. When the wavelength of the illuminating light is $910 \mathrm{~nm}$ or longer, a reflector improves sensitivity. We surmise that this result is due to a part of the back-side illumination light reaching the reflector and the resulting additional absorption of the reflected light. For the $970 \mathrm{~nm}$ light, sensitivity without the reflector is improved by a factor of 1.7 relative to front-side illumination. This is roughly consistent with the fact that the pixel opening area ratio as seen from the back-side is 1.6 times larger. With the reflector, the sensitivity is higher than front-side illumination by a factor of 2.2. Accordingly, sensitivity was improved a further $30 \%$. We surmise that this improvement is due to the greater absorption by the reflector.

\section{Conclusion}

We conducted experiments on increasing the sensitivity of image sensors that have a conventional pixel structure to near-infrared light. We fabricated a test chip with back-illuminated sensors that have a $32 \pm 4 \mu \mathrm{m}$-thick backside silicon and use the metal wiring layer as a reflector. The results of using the test device to evaluate the dependence of photosensitivity to nearinfrared light on wavelength show that for illuminating light wavelengths of $830 \mathrm{~nm}$ and above, back-side illumination produces higher sensitivity than front-side illumination. For wavelengths of $910 \mathrm{~nm}$ and above, a reflector improves sensitivity, and for $970 \mathrm{~nm}$ light, the sensitivity improvement factor is 2.2 relative to front-side illumination. The effect of the reflector is an increase in sensitivity of about $30 \%$. In this test chip, about $30 \%$ of the incident light is lost to reflection at the interface of the back-side silicon and the air. Accordingly, an anti-reflection coating on the back-side silicon can be expected to raise the sensitivity ratio to about 3. Furthermore, the wavelength of the target near-infrared light can be varied by adjusting the thickness of the back-side silicon.

\section{Acknowledgments}

This work was supported by a grant of Knowledge Cluster Initiative implemented by Ministry of Education, Culture, Sports, Science and Technology (MEXT) via Fukuoka Industry, Science \& Technology Foundation. 